\title{
Correction to: Complexity of English as a Multilingua Franca: Place of Monolingual Standard English
}

\author{
Tomokazu Ishikawa
}

\section{Correction to:}

Chapter 5 in: M. Konakahara and K. Tsuchiya (eds.), English as a Lingua Franca in Japan, https://doi.org/10.1007/978-3-030-33288-4_5

The original version of this chapter was inadvertently published with an incorrect author name in the reference list. The author's name "Wei, Li" has now been corrected to "Li, Wei". The corrections to this chapter have been updated with the changes. 